\title{
Nanoscale Light Sources for Optical Interconnects
}

\section{Li N1, Han $\mathbf{K}^{1}$, Sorger $\mathbf{V}^{2}$ and Sadana $\mathbf{D}^{1 *}$}

${ }^{1}$ IBM, TJ Watson Research Center, Yorktown Heights, NY, USA

${ }^{2}$ Department of ECE, George Washington University, Washington DC, USA

\section{Introduction}

This editorial is aimed at addressing two key aspects of nanoscale light sources: (1) low-power optical communication and (2) crystallographic defect engineering for monolithic integration with silicon. We will further discuss opportunities and challenges for nanoscale light sources for next generation, high density optical interconnect. Designing and prototyping light sources with sub light wavelength dimensions has been the topic of keen interest because of their versatility in optical communication. For example, nano light sources can operate at hundreds of $\mathrm{GHz}[1,2]$ which is not possible with conventional light sources [3]. In addition, power consumption in interconnects with these light sources can be reduced by omitting the modulator and using direct source modulation to encode optical data [4]. There are a number of nano light sources under investigation: (i) small photonic mode laser [5-9], (ii) plasmonic lasers [10,11] (iii) photonic-plasmonic hybrid lasers [12-15] and (iv) nanoscale LEDs $[16,17]$. Pros and cons of these nano light sources are discussed below.

Nano scale photonic mode lasers based on photonic crystals $(\mathrm{PhC})$ have shown the most promise requiring the lowest threshold current due to the high cavity quality factor $[8,9]$. The main drawback of such devices is their relatively large foot print $\left(\sim 10 \mu_{\mathrm{m}^{2}}\right)$ which makes them unsuitable for monolithic integration with $\mathrm{Si}$ (see discussion below). On the other hand, metal cladded plasmonic lasers which have sub light wavelength foot print are capable of achieving high optical confinement, but these suffer from optical losses in the metal. For this reason plasmonic lasers operate at higher threshold currents than what is expected from a scaled optoelectronic device [18]. As snch, the threshold current for state-of-the-art laser is in the tens of ${ }^{\mu_{A}}$ 's range for deep-sub-wavelength lasing modes [19]. Most successful demonstrations to date on plasmonic lasers have been conducted by optical pumping because electrical-pumping requires either low temperature operation or large mode size above the diffraction limit [3]. To address shortcomings of plasmonic lasers, hybrid mode photonicplasmonic lasers, whose mode primarily resides in the low-index oxide layers instead of the gain medium, have been designed. However, the low optical confinement factor of the hybridized optical mode limits the optical gain $[13,14]$. The most attractive option for a nano light source appears to be a nanoscale LED for two reasons: (1) improved Purcell factor resulting from the small cavity volume requirement $[1,20]$ and (2) no threshold current requirement. The downside of nano-LEDs is their limited power output.

\section{Challenges}

\section{Output power}

The minimum power required for a nano-LED light source should exceed the quantum shot noise limit ( 20 photons/bit) of a photodetector for optical communication to maintain a bit-error-rate of $10^{-9}$ [21]. However, the state-of-the-art photodetectors have significantly higher power requirements [22,23] which poses challenge for nanoLEDs based optical communication. Nano-photo detectors with very low detector capacitance $[24,25]$ have been proposed but such devices have not yet been successfully demonstrated.

\section{Surface passivation}

To achieve high Purcell factor and enable high modulation speeds, metal cavity devices, such as plasmonic devices require cavity dimensions on the order of $100 \mathrm{~nm}$. Highly effective surface passivation is therefore required to achieve surface recombination velocity to $<10^{4} \mathrm{~cm} / \mathrm{s}$. Although successful passivation schemes have been demonstrated [26], process compatibility and long-term stability has not yet been studied.

\section{Efficiency}

Nano plasmonic devices inherently have higher optical loss in the cavity than conventional light sources due to smaller volume. So far the demonstrated power efficiency and quantum efficiency are still orders of magnitude lower than conventional lasers. Photonic crystal lasers and nano-LEDs, are better alternatives for higher efficiency $[9,16]$.

\section{Optical coupling}

Efficient coupling into an adjacent on-chip waveguide is challenging for small light sources, due the impedance mismatch between the high wave vector mode inside the nanolaser and the lower k-vector of the waveguide. However, several groups have recently achieved improved coupling efficiency of $\sim 70 \%[7,27,28]$. This required significant simulation, design optimization, and experimental control.

\section{Reliability}

Even though the power output of a nanolight source is typically in the sub micro watt range, its power density is nevertheless higher compared to a conventional light source. The high power density/high current density may cause reliability issues, such as electro migration and device heating [29]. Additionally, reliability issues could arise due to mechanical and thermal stresses in a packaged product.

\section{Line width}

Nano lasers are prone to increased spectral line width due to enhanced Purcell factor. Therefore, such lasers may not be suitable for DWDM applications. However, CWDM application might be possible because wavelength multiplexing in this case is relaxed.

\section{Opportunities}

Nano light sources are an attractive option for highly scaled shortdistance optical interconnects [4]. We identify two key opportunities

*Corresponding author: Sadana D, IBM, TJ Watson Research Center, 1101 Kitchawan Rd, Yorktown Heights, NY 10598, USA, Tel: 914-945-3000; E-mail: dksadana@us.ibm.com

Received December 20, 2017; Accepted December 29, 2017; Published December 31, 2017

Citation: Li N, Han K, Sorger V, Sadana D (2017) Nanoscale Light Sources for Optica Interconnects. J Laser Opt Photonics 4: e114. doi: 10.4172/2469-410X.1000e114

Copyright: (c) $2017 \mathrm{Li} \mathrm{N}$, et al. This is an open-access article distributed under the terms of the Creative Commons Attribution License, which permits unrestricted use, distribution, and reproduction in any medium, provided the original author and source are credited. 
for nanolight sources: (i) monolithic integration, and (ii) three orders of magnitude higher density in optical interconnects on a semiconductor IC.

\section{Monolithic Integration on Si or SOI Substrates}

Sub-light wavelength dimensions of nanolight sources enable monolithic integration with $\mathrm{Si}$ or SOI substrates in an unprecedented manner, i.e., the integration does not degrade the LED efficiency due to lattice mismatch related crystallographic defects. This is because the probability of a dislocation or other crystallographic defect existing in the active device area decreases super linearly as devices approach nanometer dimensions (Figure 1). A monolithically grown III-V nano light source on Si substrate was indeed fabricated and its performance was analyzed [30,31]. We stipulate that when these devices are miniaturized to dimensions that are comparable to or smaller than the spacing between crystallographic defects, it may be possible to avoid defects in a majority of these devices to achieve higher performance on silicon.

\section{High density optical interconnects}

As an example, Figure 2 shows a nano-LED integrated monolithically by growing either a patterned III-V or a patterned III-N based LED structure onto an SOI substrate. A low-capacitance $(\sim 30$ $\mathrm{aF})$ and high efficiency Ge PIN photodiode is required. Numerical simulations indicate that $50 \%$ EQE is achievable with such a detector [32]. A low-loss waveguide is preferred. Previously, a "etch-less" waveguide has indeed been demonstrated earlier using SOI $(0.3 \mathrm{~dB} / \mathrm{cm})$ in conjunction with selective oxidation to obtain high quality sidewalls [33]. Near ideal transmission (up to 93\%) could be achieved over a 1 $\mathrm{cm}$ distance. For a more typical loss of $\sim 2 \mathrm{~dB} / \mathrm{cm}$, the transmission efficiency of the waveguide drops to $\sim 63 \%$ for a distance of $\sim 1 \mathrm{~cm}$, but it can maintain $\sim 95 \%$ transmission efficiency for a distance of $\sim 1 \mathrm{~mm}$.

Based on the scheme shown in Figure 2, it can be postulated that the optical interconnect density with nano-LEDs can be increased by three orders of magnitude over the current density. For example, for a waveguide with $\sim 70 \%$ coupling efficiency, a transmitter with an EQE of $\sim 20 \%$, and photon energy of $\sim 1 \mathrm{eV}, \sim 125$ photons/bit will be required to generate charge equivalent to $0.3 \mathrm{~V}$. This corresponds to energy consumption of $\sim 0.12 \mathrm{fJ} / \mathrm{bit}$ for the nano-LED. We estimate that a nano-LED with a drive current of $5 \mu_{\mathrm{A}}$ can operate at 40 $\mathrm{GHz}$ which will correspond to average optical power of $\sim 0.8{ }^{\mu_{\mathrm{W}}} \mathrm{W}$ in the waveguide. This power level is over 3 order of magnitude lower

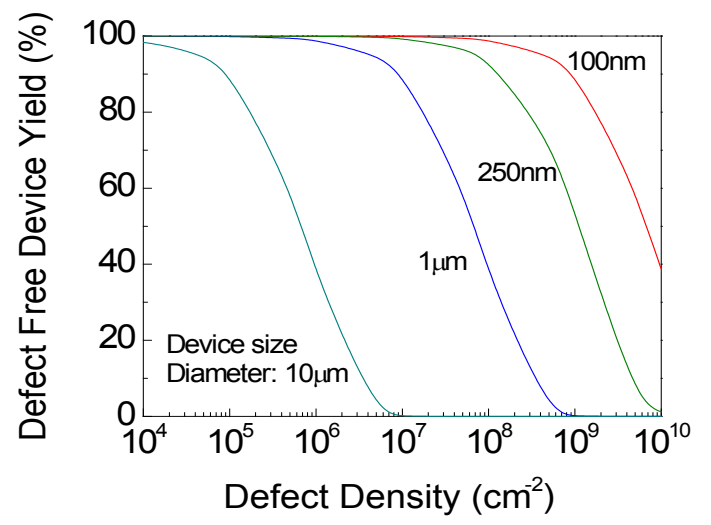

Figure 1: Defect free device yield of various sizes of devices as a function of defect density for III-V materials directly grown on Si substrate.

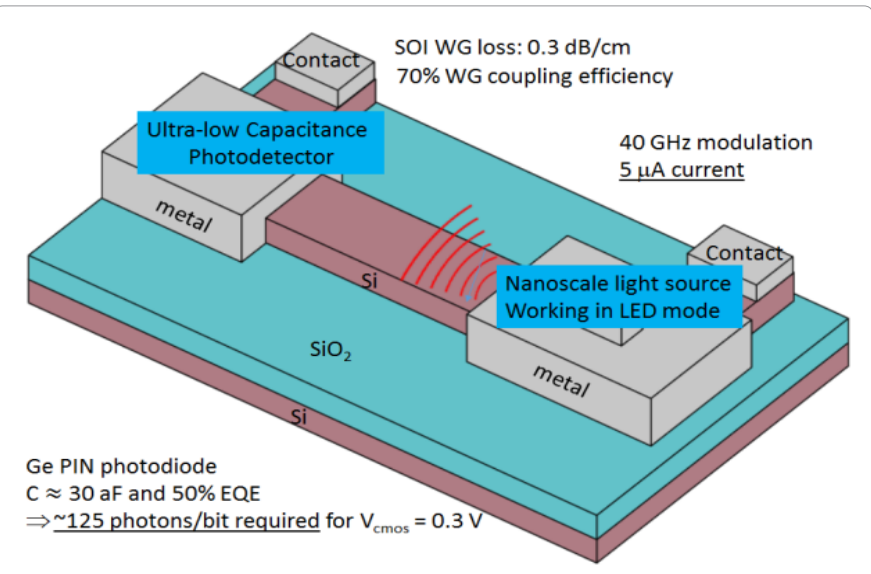

Figure 2: One possible scenario for an optical interconnect with a nanoscale light source, a low loss waveguide, and a low-capacitance photodetector.

compared to a standard conventional InP laser in use presently for optical interconnects. Therefore, nano optical devices in conjunction with monolithic integration has the potential to increase the number of chip-integrated devices from $\sim 10^{3}$ presently to $10^{6}$ in future, notwithstanding the challenges described above.

\section{Concluding Remarks}

In summary, it is clear that nano light sources are a very attractive option for future optical interconnects for high band width optical communication. The possibility that monolithic integration of these devices on Si or SOI can be done without performance degradation will create a new paradigm for on-chip optical communication at short distances $(<1 \mathrm{~cm})$. Such integration can be scaled for high volume manufacturing making it cost competitive.

\section{References}

1. Adrian Ni CY, Chuang SL, (2012) Theory of high-speed Nano lasers and nanoLEDs. Opt Express 20: 16450-16470.

2. Genov DA, Oulton RF, Bartal G, Zhang X (2011) Anomalous spectral scaling of light emission rates in low-dimensional metallic nanostructures. Phys Rev $B$ 83: 245312.

3. Ma RM, Oulton RF, Sorger VJ, Zhang X (2012) Plasmon lasers: coherent light source at molecular scales. Laser \& Photonics Rev 7: 1-21.

4. Sun S, Badaway A, Narayana V, El-Ghazawi T, Sorger VJ, (2015) PhotonicPlasmonic Hybrid Interconnects: Efficient Links with Low latency, Energy and Footprint. IEEE Photonics Journal 7: 6.

5. Ding K, Liu ZC, Yin LJ, Hill MT, Marell MJH, et al. (2012) Room-temperature continuous wave lasing in deep-sub wavelength metallic cavities under electrical injection. Phys Rev B 85: 41301.

6. Yu K, Lakhani A, Ming CWu (2010) Sub wavelength metal-optic semiconductor Nano patch lasers. Opt Express 18: 8790-8799.

7. Crosnier G, Sanchez D, Bouchoule S, Monnier P, Beaudoin G, et al. (2017) Hybrid indium phosphide-on-silicon nanolaser diode. Nat Photonics 11: 297300.

8. Ellis B, Mayer MA, Shambat G, Sarmiento T, Harris J, et al. (2011) Ultralowthreshold electrically pumped quantum-dot photonic-crystal nanocavity laser. Nat. Photonics 5: 297-300.

9. Takeda K, Sato T, Shinya A, Nozaki K, Kobayashi W, et. al. (2013) Few-fJ/bit data transmissions using directly modulated lambda-scale embedded active region photonic-crystal lasers. Nat Photonics 7: 569-575.

10. Khajavikhan M, Simic A, Katz M, Lee JH, Slutsky B, et al. (2012) Threshold less nanoscale coaxial lasers. Nature 482: 204-207.

11. Hill MT, Marell M, SP Leong E, Smalbrugge B, Zhu Y, et al. (2009) Lasing in 
Citation: Li N, Han K, Sorger V, Sadana D (2017) Nanoscale Light Sources for Optical Interconnects. J Laser Opt Photonics 4: e114. doi: 10.4172/2469-410X.1000e114

Page 3 of 3

metal-insulator-metal sub-wavelength plasmonic waveguides. Opt Express 17: 11107-11112.

12. Wei W, Yan X, Zhang X (2016) Ultrahigh Purcell factor in low-threshold Nano laser based on asymmetric hybrid plasmonic cavity. Sci Rep 6: 33063.

13. Oulton RF, Sorger VJ, Zentgraf T, Ma RM, et al. (2009) Plasmon lasers at deep sub wavelength scale. Nature 461: 629-632.

14. Ma RM, Oulton RF, Sorger VJ, Bartal G, Zhang X (2011) Room-temperature sub-diffraction-limited plasmon laser by total internal reflection. Nat Mater 10: 110-113

15. Liu N, Gocalinska A, Justice J, Gity F, Povey I, et al. (2016) Lithographically Defined, Room Temperature Low Threshold Subwavelength Red-Emitting Hybrid Plasmonic Lasers. Nano Lett 16: 7822-7828.

16. Fortuna SA, Taghizadeh A, Yablonovitch E, Wu MC, (2016) Toward 100 $\mathrm{GHz}$ direct modulation rate of antenna coupled nanoLED. IEEE Photonics Conference, pp: 216-217.

17. Dolores-Calzadilla V, Romeira B, Pagliano F, Birindelli S, Higuera-Rodriguez A, et al. (2017) Waveguide-coupled nanopillar metal-cavity light-emitting diodes on silicon. Nat Commun vol: 8 .

18. Liu K, Sun S, Majumdar A, Sorger VJ (2016) Fundamental Scaling Laws in Nanophotonics. Nature: Scientific Reports 6: 37419.

19. https://www.degruyter.com/view/j/nanoph.2012.1.issue-1/nanoph-2012-0017/ nanoph-2012-0017.xml

20. Lau EK, Lakhani A, Tucker RS, Wu MC (2009) Enhanced modulation bandwidth of Nano cavity light emitting devices. Opt Express 17: 7790-7799.

21. Papannareddy (2004) Lightwave Communication Systems: A Practical Perspective

22. DeRose CT, Trotter DC, Zortman WA, Starbuck AL, Fisheret M, et al. (2011)
Ultra compact $45 \mathrm{GHz}$ CMOS compatible Germanium waveguide photodiode with low dark current. Opt Express 19: 24897-24904.

23. Nozaki K, Matsuo S, Fujii T, Takeda K, Ono M et al. (2016) Photonic-crysta nano-photodetector with ultrasmall capacitance for on-chip light-to-voltage conversion without an amplifier. Optica 3: 483-492.

24. Tang L, Kocabas SE, Latif S, Okyay AK, Ly-Gagnon DS et al. (2008) Nanometrescale germanium photodetector enhanced by a near-infrared dipole antenna. Nat. Photonics 2: 226-229.

25. Hasan M, Simpson JJ (2013) Photonic nanojet-enhanced nanometer-scale germanium photodiode. Appl Opt 52: 5420-5425.

26. Higuera-Rodriguez A, Romeira B, Birindelli S, Black LE, Smalbrugge E, et al. (2017) Ultralow Surface Recombination Velocity in Passivated InGaAs/InP Nanopillars. Nano Lett 17: 2627-2633.

27. Kim MK, Lakhani AM, Wu MC, (2011) Efficient waveguide-coupling of metalclad nanolaser cavities. Opt Express 19: 23504-23512.

28. Kim MK, Li Z, Huang K, Going R, Wu MC, et al. (2013) Engineering of metalclad optical Nano cavity to optimize coupling with integrated waveguides. Opt Express 21: 25796-25804.

29. Tiku S, Biswas D (2016) III-V Integrated Circuit Fabrication Technology: Fabrication, Integration and Applications. CRC Press.

30. Li N, Liu K, Sorger VJ, Sadana DK, (2015) Monolithic III-V on Silicon Plasmonic Nanolaser Structure for Optical Interconnects. Sci Rep Vol: 5

31. Liu K, Li N, Sadana DK, Sorger VJ, (2016) Integrated Nanocavity Plasmon Light Sources for On-Chip Optical Interconnects. ACS Photonics 3: 233-242.

32. Going R (2015) Ultra-low energy photoreceivers for optical interconnects EECS Department.

33. Cardenas J, Poitras CB, Robinson JT, Preston K, Chen L, et al. (2009) Low loss etchless silicon photonic waveguides. Opt Express 17: 4752-4757. 